\title{
AS SAFIRAS NOS DEPÓSITOS DE DIAMANTE ALUVIONAR DA REGIÃO DO RIO COXIM, MATO GROSSO DO SUL, BRASIL
}

\author{
ANTONIO LICCARDO ${ }^{1} \&$ JOÃO EDUARDO ADDAD ${ }^{2}$
}

\begin{abstract}
RESUMO Depósitos aluvionares diamantíferos de Coxim, Mato Grosso do sul, contêm grãos de coríndon, variedade safira, em associação com os diamantes. Estas safiras foram analisadas por microssonda eletrônica para $\mathrm{Al}, \mathrm{Fe}, \mathrm{Ti}, \mathrm{Cr}$ e $\mathrm{Ga}$. A relação $\mathrm{Cr} / \mathrm{Ga}<\mathrm{I}$ indica fontes de álcali basaltos para o coríndon, pois as safiras de Coxim apresentam valores abaixo de 1 . Teores elevados de ferro, entre 1,03\% e 1,26\%, e Ti baixo, de $0,00 \%$ a $0,31 \%$, sugerem um mecanismo de cor por transferência de carga entre $\mathrm{Fe}^{2+}$ e Fe ${ }^{3+}$, típico para safiras basálticas. A presença de coríndon com características de álcali basaltos indica a possibilidade de existência desta litologia entre as rochas alcalinas da borda da Bacia do Paraná ou nas litologias fonte para sedimentos, como a Formação Aquidauana. Resistência elevada a ataques químicos, dureza e densidade de ambos o coríndon e o diamante, apontam para uma sobrevivência ao retrabalhamento sedimentar e uma convergência deposicional em armadilhas fluviais. $\mathrm{O}$ alto grau de arredondamento das safiras de Coxim indica um vetor de transporte de longa distância.
\end{abstract}

Palavras-chave: coríndon, diamante, basaltos alcalinos, Coxim

ABSTRACT SAPPHIRE IN DIAMOND ALLUVIONAR DEPOSITS AT COXIM RIVER REGION, MATO GROSSO DO SUL, BRASIL Alluvionar diamantiferous deposits of Coxim, Mato Grosso do Sul contain corundum grains, sapphire type, in association with diamonds. These sapphires were analyzed at a electron microprobe to $\mathrm{Al}, \mathrm{Fe}, \mathrm{Ti}, \mathrm{Cr}$ and $\mathrm{Ga}$. The relation $\mathrm{Cr} / \mathrm{Ga}<\mathrm{l}$ indicates alkaline basaltic source to the corundum and the Coxim sapphires presented values around $0,25-0,5$. High iron content, between $1,03 \%$ and $1,26 \%$, and low Ti, from $0,0 \%$ to $0,31 \%$, suggests cause of color by charge transfer between $\mathrm{Fe}^{2+}$ and $\mathrm{Fe}^{3+}$, typical to basaltic sapphires. The presence of alkaline basalts corundum aim the existence of this litology among the alkaline rocks from the Paraná Basin border or at the source litologies to sediments, as the Aquidauana Formation. High levels of chemical attack resistance, hardness and density of both corundum and diamond, point to a sedimentary reworking survival and a depositional convergence at fluvial traps. The high roundness of thesapphires from Coxim indicates a long distance vector.

Keywords: corundum, diamond, alcaline basalts, Coxim

Nos depósitos aluvionares dos rios Coxim, Jauru, Taquari e Piquiri, assim como de alguns de seus afluentes, Mato Grosso do Sul, Brasil, são encontrados grãos de coríndon, variedade safira, associados ao diamante. Esta associação se distribui em alguns trechos fluviais por uma faixa de aproximadamente 110 quilômetros de comprimento no sentido N-S, por 40 quilômetros, W-E (a área de ocorrência situa-se entre as coordenadas $54^{\circ} 40^{\prime}$ e $54^{\circ} 20^{\prime}, 17^{\circ} 50^{\prime}$ e $\left.18^{\circ} 55^{\prime}\right)$. Grãos de safira recuperados por garimpeiros nas catas diamantíferas apresentam tamanhos entre 1 e 12 milímetros (Fig. 1). A grande parte dos clastos de safira mostra baixo grau de euedrismo, baixa esfericidade, médio a alto arredondamento, sendo os grãos geralmente irregulares, condicionados por fraturamento (Fig. 2). Estas safiras exibem cores que variam entre acinzentado, azul, verde, castanho, amarelo, incolor e, raramente violeta. Muitos grãos exibem zoneamento de cor e inclusões, usualmente em faixas paralelamente dispostas ou concêntricas (Fig. 3 e 4). São comuns os zoneamentos azul - incolor, verde amarelo e castanho - azul. Amostras destes grãos de safira foram analisadas por microssonda eletrônica, nos modos EDS e/ ou WDS, modelos JEOL - JXA8900R no Laboratório de Microanálises, Instituto de Ciências Exatas, UFMG e CAMEBAX SX 50, no Instituto de Geociências da UnB. A Tabela 1 mostra os resultados obtidos na análise de cinco amostras de coloração azul obtidas nos concentrados diamantíferos da região.
Foram considerados os elementos alumínio, ferro, titânio, cromo e gálio, na forma de porcentagem em peso de óxidos. A relação cromo/gálio fornece indicação da origem basáltica alcalina para o coríndon, quando é claramente menor que 1 , e origem metamórfica quando fica acima deste valor, conforme os estudos de Sutherland et al.(1998), Schwartz \& Stern (2001) e Sutherland \& Schwartz (2000), para as safiras da Austrália e conforme resultados preliminares de Liccardo et al.(1999) para outras ocorrências no Brasil. Nas safiras de Coxim, a relação $\mathrm{Cr} / \mathrm{Ga}$ apresentou valores ao redor de 0,25 - 0,5 , indicando uma origem não-metamórfica, mas de basaltos alcalinos. Tanto a porcentagem alta de Fe, entre $1,03 \%$ e $1,26 \%$ em peso de óxidos, quanto a extremante baixa de Ti, variando de $0,0 \%$ (não detectado) a $0,31 \%$, também sugerem como o mecanismo causador de cor nestas safiras, a transferência de carga entre $\mathrm{Fe}^{2+}$ e $\mathrm{Fe}^{3+}$, típico de safiras encontradas em terrenos basálticos (Smith et al. 1995) em concordância com a presença de quantidades relativamente maiores de $\mathrm{Ga}$. Coríndon encontrado nos basaltos alcalinos tem sua origem na crosta inferior, sendo estas extrusivas apenas transportadores destes xenocristais (Guo et al., 1992), de maneira semelhante aos diamantes em rochas kimberlíticas. A presença de coríndon característico de álcali-basaltos nesta região sugere a existência desta litologia específica, ou de uma extrusiva que tenha desempenhado um papel semelhante, entre as alcalinas da borda da bacia do Paraná ou entre as litologias-fonte para

I Departamento de Geologia, UFOP - Campus Morro do Cruzeiro s/n, MG, 35400-000 - E-mail: liccardo@degeo.ufop.br

2 Departamento de Ecologia e Recursos Naturais, UFES - Av Fernando Ferrari s/n, Goiabeiras, Vitória, ES, 29460-910 - E-Mail: eaddad@ @otmail.com 
pacotes sedimentares como a Formação Aquidauana. Os graus elevados de resistência a ataques químicos (intemperismo), dureza e densidade de ambos os minerais, diamante e coríndon, determinam a sua sobrevivência a retrabalhamentos sedimentares sucessivos e uma convergência deposicional em armadilhas fluviais, principalmente, determinando o coríndon como acompanhante do diamante aluvionar na área em questão. Entretanto, o coríndon, de modo diferente do diamante, apresenta a sua morfologia e textura superficial como indicadoras de transporte, por ser passível de abrasão, $\mathrm{O}$ alto grau de arredondamento alcançado pelas safiras de Coxim indica um transporte de longa distância e/ou condicionante de solicitação mecânica intensa, ou ainda uma sucessão de retrabalhamentos. Esta característica dificulta a comparação da amplitude de transporte destes dois minerais e a possibilidade de inferência de uma relação parental entre as sua proveniências.

Tabela 1 - Resultados de análise química do coríndon, obtidos por microssonda eletrônica (\% em peso).

\begin{tabular}{|c|c|c|c|c|c|}
\hline Óxidos & COX-1 & COX-2 & COX-3 & COX-4 & COX-5 \\
\hline${ }^{*} \mathrm{Fe}_{2} \mathbf{O}_{3}$ & 1,06 & 1,23 & 1,07 & 1,03 & 1,26 \\
\hline $\mathrm{TiO}_{2}$ & 0,01 & 0 & 0,03 & 0 & 0,31 \\
\hline $\mathrm{Cr}_{2} \mathrm{O}_{3}$ & 0,02 & 0 & 0,02 & 0 & 0,01 \\
\hline $\mathrm{Ga}_{2} \mathbf{O}_{3}$ & 0,04 & 0,03 & 0,05 & 0 & 0,04 \\
\hline $\mathbf{A l}_{2} \mathbf{O}_{3}$ & 98,77 & 98,27 & 99,10 & 98,11 & 98,05 \\
\hline
\end{tabular}

* Considerado como Fe total

\section{Referências}

Guo J.F., O'Reilly, S.Y., Griffin W.L. 1992. Origin of sapphire in estern australian basalts, inferred from inclusion studies. Journal of Geological Society of Australia, 32: 219-220.

Liccardo, A, Jordt-Evangelista, H., Ferreira, C.M., Sabioni A.C.S., Chodur, N.L. 1999. Geoquímica em Amostras de Coríndon no Brasil. In: SBGq, Congresso de Geoquímica dos Países de Língua Portuguesa \& Congresso Brasileiro de Geoquímica, 5, Porto Seguro, Bahia, Anais, p. 274.

Schwartz, D. \& Stern, W.B. 2000. Chemical Fingerprinting as a tool for the Characterisation of Gem Corundums from different genetic Environments. In: IUGS, International Geological Congress, 31, Rio de Janeiro, CDROM.
Smith, C.P., Kammerling R.C., Keller A., Peretti A., Scarratt K.V., Khoa N.G., Repetto, S. 1995. Sapphires from Southern Vietnam. Gems \& Gemology, 31:168-186

Sutherland, F.L., Schwartz, D., Jobbins, E.A., Coenraads, R.R., Webb,G. 1998. Distinctive gem corundum suites from discrete basalt fields: a comparative study of Barrington, Australia and West Pailin, Cambodia gemfields. Journal of Gemmology, 26: 65-85.

Sutherland, F.L. \& Schwartz, D. 2001. Origin of gem corundums from basaltic fields. The Australian Gemmologist, 21:30-33

Manuscrito NB-27

Recebido em 15 de novembro de 2001 Revisào dos autores em 05 de dezembro de 200 Revisão aceita em 07 de dezembro de 2001 


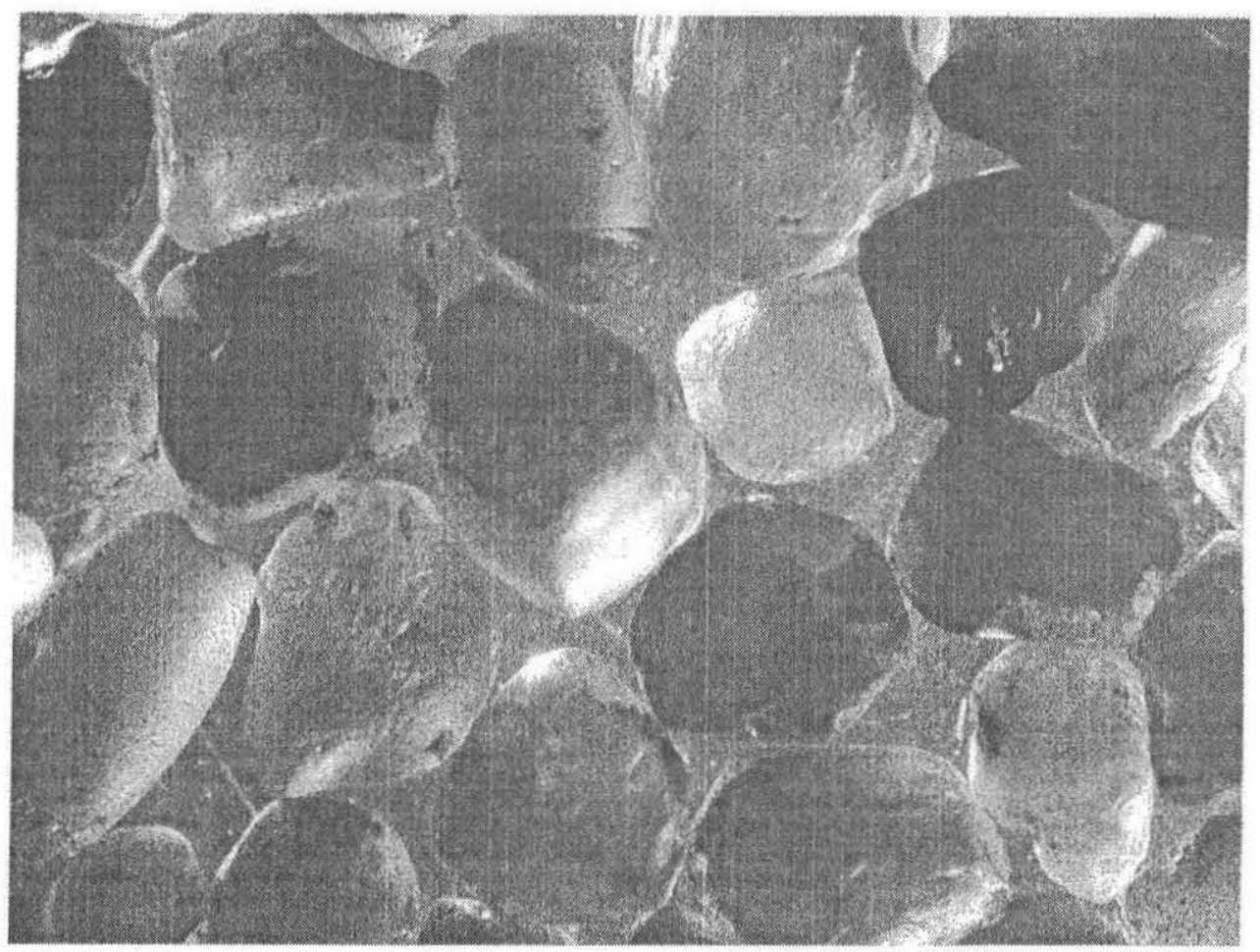

Figura I - Clastos de coríndon, variedade safira, de concentrados diamantíferos do rio Coxim, MS.

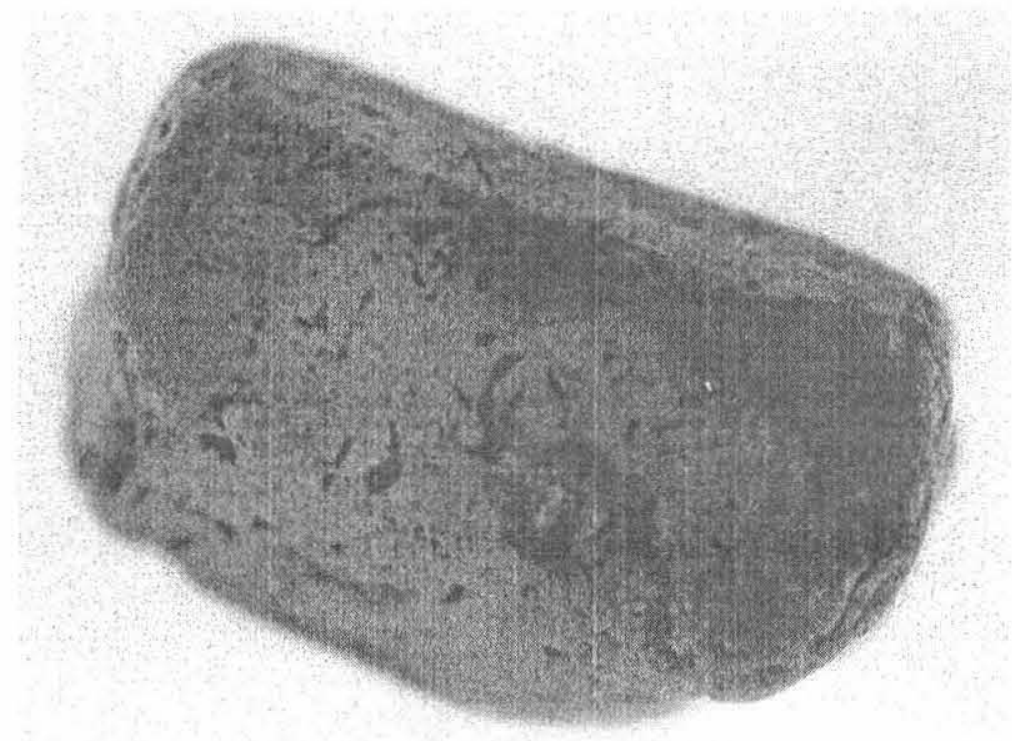

Figura 2 - Grão de safira com grau médio de euedrismo, formato "em barril", de concentrados diamantíferos do rio Coxim, $M S$. 


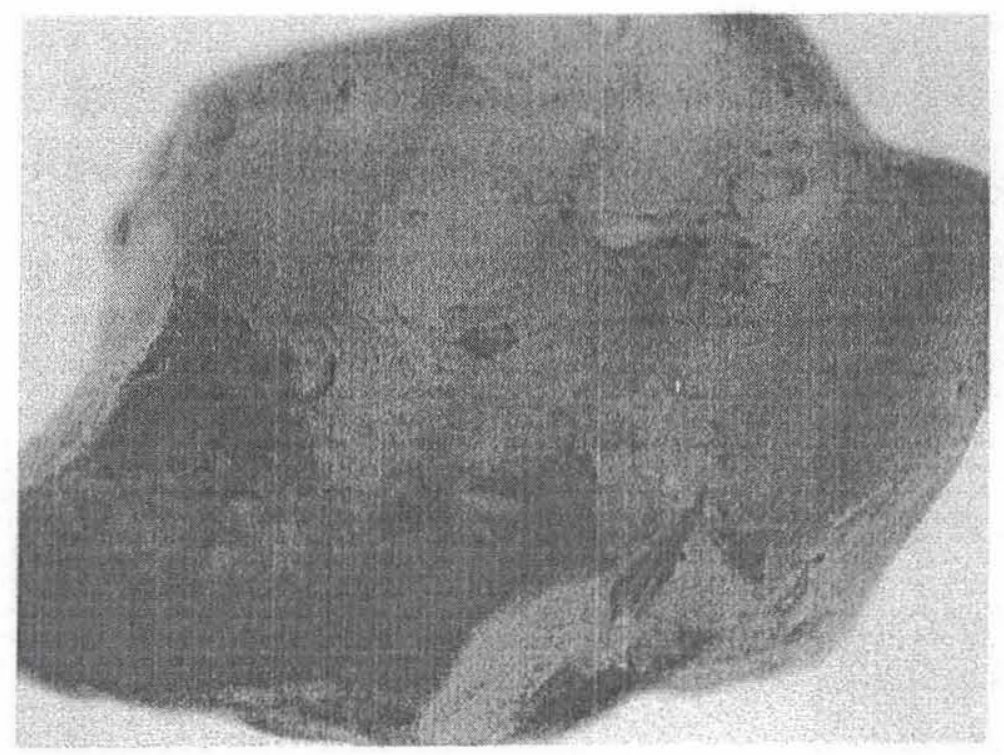

Figura 3 - Grãos de safira com zonação de cor em faixas, de concentrados diamantíferos do rio Coxim, MS.

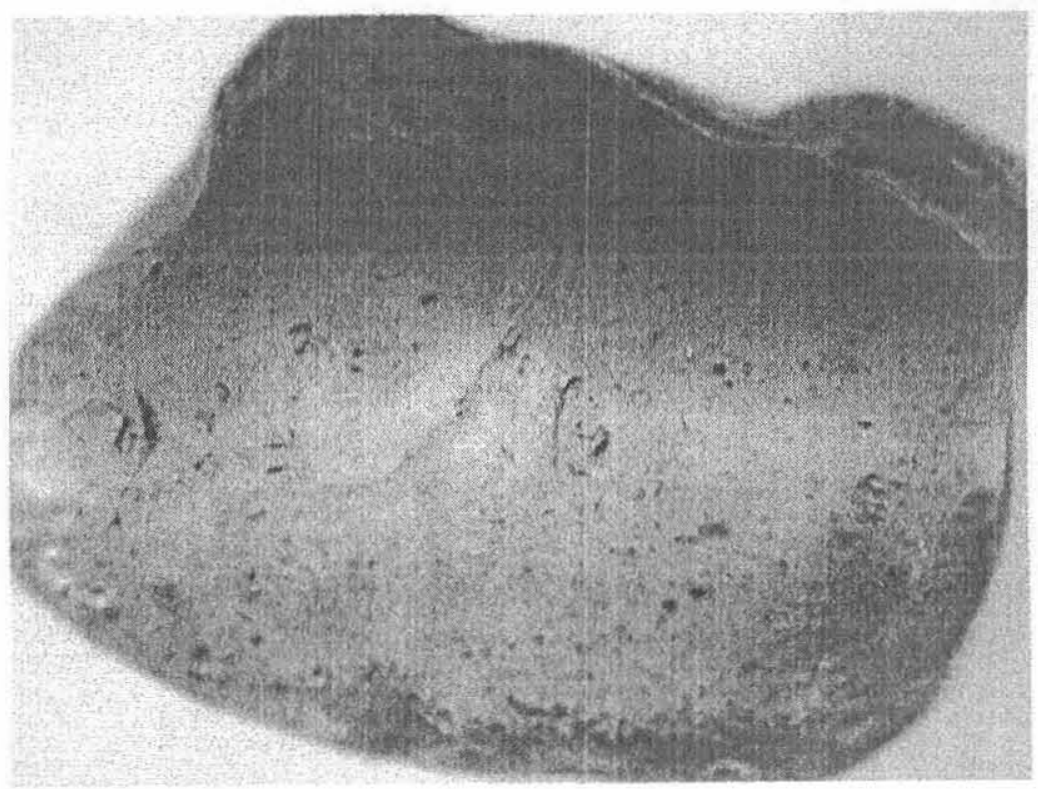

Figura 4 - Grãos de safira com zonação de cor em faixas, de concentrados diamantíferos do rio Coxim, MS. 